\title{
Event Related Biometrics: Towards an Unobtrusive Sensing Seat System for Continuous Human Authentication
}

\author{
Marcello Ferro $^{1, *}$, Giovanni Pioggia ${ }^{2}$, Member, IEEE, Alessandro Tognetti ${ }^{1}$, Gabriele Dalle Mura ${ }^{1}$ and \\ Danilo De Rossi ${ }^{1}$
}

\author{
${ }^{1}$ Interdepartmental Research Center "E. Piaggio" \\ Faculty of Engineering, University of Pisa, Italy \\ ${ }^{2}$ Institute of Clinical Physiology (IFC) \\ National Council of Research (CNR) of Pisa, Italy
}

\begin{abstract}
The present work is focused on the improvement of a Sensing Seat system previously developed by the authors for the initial authentication purpose in office and car scenarios. The goal is to obtain an event-related continuous authentication system, where the human subject should not take care of the system itself so that he is free to perform his normal actions. The system is realized by means of a sensing cover where conductive elastomers are used as strain sensors. The deformation of the cover caused by the body shape while actions are performed by the subject are used to obtain time-dependent relevant features. Such information are then analyzed by suitable classifiers that are able to perform the real-time continuous authentication task. A measurement campaign was carried out using data from 24 human subjects employed in an office scenario while a set of 22 actions were performed. The authentication capabilities of the system are reported in terms of acceptance and rejection rates, showing a high degree of correct classification.
\end{abstract}

Index Terms-strain sensor, sensing seat, event-related human authentication, monitoring, security, biometrics

\section{INTRODUCTION}

The use of biometrics for access control in restricted infrastructures has been very extensively researched during the last four decades. The present work focused on the development of a Sensing Seat system for real-time event-related continuous human authentication purpose.

Several companies are currently working in order to realize comfortable interactive seats. These systems, some of them already on the market [1], [2], [3], [4], use different technologies and materials, but they share the use of sensors that measure the pressure exerted on the seat by the passenger. Actually, no existing system based on sensing seat is able to perform the human authentication task and no result on this topic, even if in a preliminary stage, was found in literature review. The existing systems, some of them are reported below, are more focused on comfort monitoring, pressure mapping, air-bag activation and event-related tasks. However, the information

*Corresponding author: Dr. Marcello Ferro, Ph.D. Interdepartmental Research Center "E. Piaggio", Faculty of Engineering, University of Pisa, Via Diotisalvi 2, 56100 Pisa, Italy. E-mail: marcello.ferro@ing.unipi.it supplied by these systems may be used to extract features useful for the authentication task. Additionally, patents [5], [6], [7] and academy works [8], [9], [10] were found on this topic. For many systems it was not possible to find accurate test results in terms of classification capabilities in biometrics tasks.

In this work, the authors show the design and realization of an unobtrusive and versatile sensing seat system for human authentication that can be employed in different scenarios such as truck and car pilots, airplane pilots, plant and office personnel, and, in general, environments where the security is mandatory and a soft seat is available.

A Sensing Seat system [11] was initially developed by the authors, where the enrollment and the authentication procedures were carried out with the cooperation of the user, according to the instructions supplied by the system. The mentioned prototype is able to supply a one-dimensional deformation profile, and, after a feature extraction process, the system performs the initial human authentication task. A new prototype is being developed to obtain a continuous authentication, without interfering with the user actions and according to the detection of predefined events. According to this purpose, the previous Sensing Seat prototype was upgraded and a new control system was developed to handle the issues regarding the continuous authentication as well as the event notifications of an high-level core system. The new prototype was tested during a measurement campaign in a smart room environment equipped with 5 cameras: 29 subjects were analyzed in an office scenario, over 2 sessions consisting in 6 repetitions each. A total of 22 actions were taken into account. The video streams were subsequently analyzed to obtain the annotation data to simulate the event notification mechanism. The test results show an high recognition degree, suggesting that, potentially, a more complex hardware system may result in more reliable results. According to this, the authors designed and realized a new Sensing Seat hardware system, where sensor information can supply data about the two-dimensional seat cover deformation. As the new prototype will be ready, a new measurement campaign will be conducted and the event-related authentication results will be compared to the ones obtained with the previous prototype.

The proposed system, as part of the ACTIBIO project for multi-modal event-related human authentication [12], has been preliminarily tested in simulated office scenario and it will be tested in office and car pilots. ACTIBIO aims to research and 
develop a completely new concept in biometric authentication, that is the extraction of biometric signatures based on the response of the user to specific stimuli while performing specific work-related activities. The novelty of the approach lies in the fact that the measurements that will be used for authentication will correspond to the response of the person to specific events being however, fully unobtrusive and also fully integrated in an ambient intelligence infrastructure.

\section{Sensing System}

The sensors developed by at the University of Pisa allow piezoresistive sensing fabrics to be realized [13], [14]. Mixtures of polymers deepened with coal directly printed onto the fabric allow strain to be transducted. The strain sensors are realized by means of Conductive Elastomers (CE) composites. CE composites show piezoresistive properties when a deformation is applied and can be easily integrated into fabric or other flexible substrate to be employed as strain sensors (Fig.2, 1) . The CE we used is based on a WACKER Ltd (Elastosil LR $3162 \mathrm{~A} / \mathrm{B}$ ) product [15]. It consists in a mixture of graphite and silicon rubber. WACKER Ltd guarantees the non-toxicity of the product. The sensing layer consists in several arrays of strain sensors directly printed into a Lycra tissue. An electronic interface manages, besides the prefiltering, the digitizing of the signal and the communication with the personal computer. Because of the nature of the adopted sensors and the possibility to cover the surfaces with a redundant net of them, we could choose the maximum number of the sensors for covering uniformly the surfaces of contact. The resulting design results in a high-impedance circuitry where a reference current is injected. The high-impedance characteristic allows both sensors and wires to be realized by means of the same technology and to gain unobtrusivity. Indeed, the use of common electrical wires is avoided within the sensing layer. Moreover the power consumption is quite zero resulting in a completely safe system. A connector plug is placed in one side of the sensing cover in order to connect the system to the front-end module. The sensing layer is able to respond to simultaneous deformations in different directions by means of a piezoresistive network which consists of CEs composite rubbers screen printed onto a cotton Lycra fabric. They are elastic and do not modify the mechanical behavior of the fabric. In the production process of sensing fabrics, a solution of CE and trichloroethylene is smeared on a Lycra substrate previously covered by an adhesive mask. The mask is designed according to the desired topology of the sensor network and cut by a laser milling machine. After the deposition, the cross-linking process of the mixture is obtained at a temperature of $130^{\circ} \mathrm{C}$. Furthermore, by using this technology, both sensors and interconnection wires can be smeared by using the same material in a single printing and manufacturing process.

The existing Sensing Seat prototype was adapted to the event-related strategy discussed in this work. The system includes 32 strain sensors and a new cover was realized specifically for an office seat (Fig. 3).

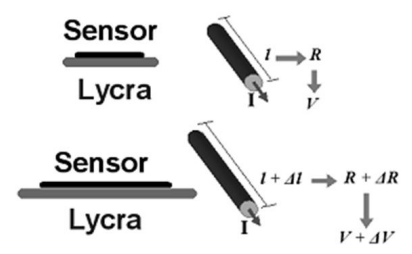

Figure 1. Transduction principle of the conductive elastomer strain sensor

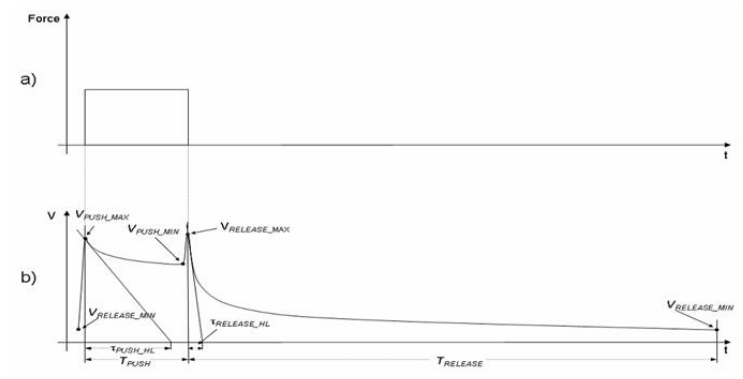

Figure 2. Strain sensor voltage signal and extracted features (b) in response to a strain impulse (a)

\section{DATA ACQUisition System}

The relevant block schemas for the Sensing Seat system are shown in Fig. 4. The sensing cover is connected to a front-end conditioning module that performs a high-impedance voltage reading of the $\mathrm{CE}$ strain sensors. Data are sent to a data acquisition board (National Instruments DAQ) that is responsible of the raw data dispatching to the client-side module. The system provides a complete voltage information of the 32 sensor nodes with a sampling rate of $100 \mathrm{~Hz}$. The voltage resolution is of 1 millivolt in the range from 0 to 5 volts. The system is connected to the client-side PC via an USB 2.0 connection. The real-time data acquisition and analysis are performed using a suitable software framework for sensory fusion [16] and biometric data management [17].

a)

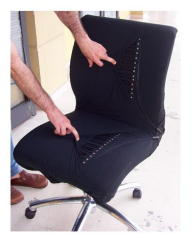

Figure 3. The Sensing Seat system prototype: a) The office seat equipped with the sensing cover; b) Details of the sensor connections on the bottom side of the sensing cover 


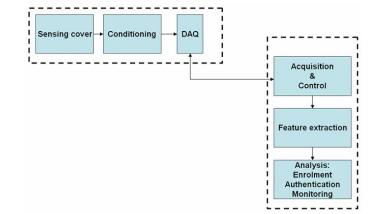

a)

Figure 4. The Sensing Seat system block schema: a) Logical schema; b) physical schema and relationship between the high level core system

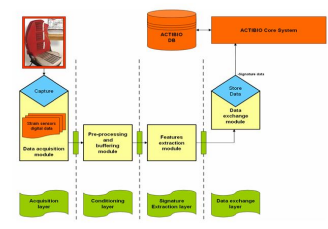

a)

b)

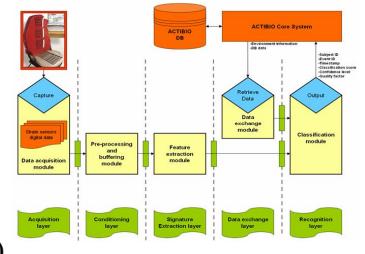

Figure 5. The Sensing Seat system recording protocol: a) enrollment task b) authentication and monitoring tasks

\section{SOFTWARE ARCHITECTURE}

Once the system is initialized, the raw data are continuously acquired, a low-pass filter is applied and resulting data are stored in a memory buffer whose length is configurable, depending on the maximum delay that is expected from the event-detection mechanism of the high-level core system. During the enrollment and the authentication task, the system receives the event notification from the core system and, for each received event, the system retrieves the raw sensor data from the memory buffer belonging to the event-related begin and end timestamp information.

As the event-related time window is identified, the raw data are used to extract the relevant features. The following features are extracted:

- min, max and mean values for each sensor over time, obtaining 3 curves over the sensor space

- 5 polynomial coefficients are evaluated (with Least Squares) for each of the 3 curves

During the enrollment task, the features are associated to the specific event identifier and to the specific subject identifier so that the corresponding signature is updated [18], [19]. During the authentication task the features are associated to the action identifier and the resulting signature is compared to the one stored during the enrollment step.

The authentication task is performed by a personal and event-related classifier that was trained on the basis of the signature acquired during enrollment. At training time, the optimal threshold if found minimizing the equal error rate (EER) as the best trade-off between the false acceptance rate (FAR) and the false rejection rate (FRR). Several classifiers were taken into account [20], [21], [22]:

- Principal component analysis (PCA)

- Multilayer perceptron (MLP)

- Kohonen self-organizing map (KSOM)

- Probabilistic neural network (PNN)

- Vector distance classifier (Euclidean distance) (VDC)

\begin{tabular}{cc}
\hline Action ID & Action description \\
\hline 1 & Phone conversation \\
2 & Phone conversation (light) \\
3 & Phone reached the ear \\
4 & Phone left from ear \\
5 & Interacting with mouse \\
6 & Interacting with mouse (light) \\
7 & Writetyping \\
8 & Writetyping (light) \\
9 & Writing with pencil \\
10 & Writing with pencil (light) \\
11 & Talking to the microphone panel \\
12 & Talking the microphone panel (light) \\
13 & Pressing buttons in the office panel \\
14 & Pressing buttons in the office panel (light) \\
15 & Drinking from glass \\
16 & Drinking from glass (light) \\
17 & Filling glass with water \\
18 & Yawning \\
19 & Raising Hands \\
20 & User is seated \\
21 & Watching video \\
22 & No activity \\
\hline
\end{tabular}

Table I

LIST OF THE ACTIONS TAKEN INTO ACCOUNT DURING THE MEASUREMENT CAMPAIGN. THE "LIGHT" ACTIONS INDICATES A LOW RELIABLE DETECTION OF THE RELATED EVENT.

\section{Measurement Campaign and Results}

The Sensing Seat system was tested during a measurement campaign in a smart room environment equipped with 5 cameras: 29 subjects were analyzed in an office scenario, over 2 sessions consisting in 6 repetitions each. The duration of a single recording was of 10 minutes and a total of 22 actions were taken into account. The video streams were subsequently analyzed to obtain the annotation data of the actions in order to simulate the event notification mechanism. The recordings gave the opportunity to have real data to be used to evaluate the system authentication performances. During the recordings all the modules (the Camera Servers and the Sensing Seat server system), were synchronized using a unique clock reference. As a result, all raw data were acquired using a timestamp where the synchronization error was always less than 10 milliseconds. The list of the actions for the event triggering are show in Table I.

The classifiers were trained on $80 \%$ of the available examples for each action and subject, while the remaining $20 \%$ was used as the test set. The better results were supplied by the VDC classifier and the results in term of FAR and FRR for each action and for each subject are shown in Fig. 6. The maximum FAR is $4 \%$ while the maximum FRR is $0.5 \%$, showing that the Sensing Seat system may be successfully employed in continuous event-related authentication scenarios.

\section{CONCLuSiOns}

In this paper the development of a novel Sensing Seat system based on an unobtrusive piezoresistive sensor array is described. The main result is a positive assessment of the realtime continuous authentication task, showing the robustness of the system in terms of biometric rates. Another relevant result is the assessment of the strain sensor technology and 

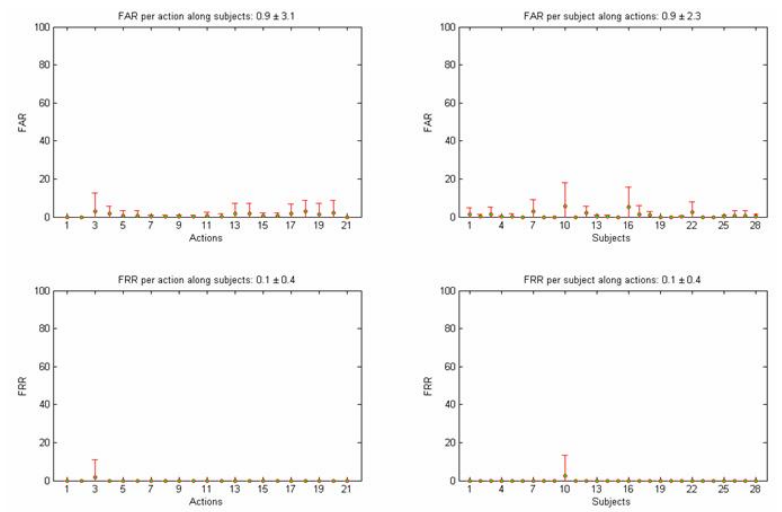

Figure 6. Authentication error: mean and standard deviation of FAR (top) and FRR (bottom) along subjects (left) and actions (right)

of the classification modules based on personal and eventrelated classifiers. The modular software library compliant with BioAPI makes the sensing seat system able to act in different scenarios and to be employed together and in cooperation with other authentication systems.

The proposed system is still under development even if the actual prototype was successfully tested within unimodal and multimodal environments. The main advantage in respect of the previous Sensing Seat prototype is represented by the absence of the cooperation of the human subject during the monitoring stage. The open issues include the performance study in extreme environmental conditions (e.g. very low and very high environmental temperature scenarios). Moreover, the strain sensor stability over time as well as its chemical properties must be investigated thoroughly in order to study the sensor degeneration over time (i.e. sensor aging). Additionally, in order to make the system really unobtrusive, the objects inside the clothes and the pockets (e.g. keys, wallet) should be treated as a point of disturbance to increase the final user convenience. All the above mentioned topics will be taken into account in future developments.

\section{ACKNOWLEDGMENT}

The present work is part of the ACTIBIO project within the Seventh Framework Programme, Theme 3, Information Society Technologies, Contract Number 215372. The authors wish to thank the entire ACTIBIO consortium and in particular the Universitat Politècnica de Catalunya (UPC) partner that supplied the smart room where the simulated office recordings where carried out to perform the analysis and the tests presented in this work.

\section{REFERENCES}

[1] The Tekscan Company, http://www.tekscan.com/industrial/app_seating. html, last accessed on 17/03/2008.

[2] The Novel Company, http://www.novel.de/, last accessed on 17/03/2008.

[3] The Softswitch Company, http://www.softswitch.co.uk/, last accessed on 17/03/2008.
[4] The Johnson Controls Company, http://www.johnsoncontrols.com/, last accessed on 17/03/2008.

[5] FORD GLOBAL TECHNOLOGIES INC., "Vehicle air bag deployment dependent on sensing seat and pedal positions", patent, Priorities: [US09681903 22 Jun 2001], UKC Headings: G4N Int Cl7 B60R 21/01, B60R 21/16, GB2377536 (GB0212617.5), May 2002.

[6] Hilliard G. G., "Seat cushion", patent, GB0228513.8, December 2002.

[7] Federspiel L., "Sensor mat for a vehicle seat", I.E.E. International Electronics \& Engineering S.a.r.l., patent, US6794590, September 2004.

[8] Tan H. Z., Slivovsky L. A., Pentland A., "A sensing chair using pressure distribution sensors", IEEE/ASME Transactions on Mechatronics, Vol. 6, No. 3, pp. 261-268, 2001.

[9] S. Mota and R. W. Picard, "Automated posture analysis for detecting learner's interest level" in Workshop on Computer Vision and Pattern Recognition for Human-Computer Interaction, CVPRW'03, ISSN 10636919, ISBN 0-7695-1900-8, vol. 5, page 49, Madison, Wisconsin, USA, June, 2003

[10] Rosen J., Arcan M., "Modeling the Human Body/Seat System in a Vibration Environment", Journal of Biomechanical Engineering, Volume 125, Issue 2, pp. 223-231, April 2003

[11] EC co-funded Specific Targeted Research Project (STREP), "Human Monitoring and Authentication Using Biodynamic Indicators and Behavioral Analysis (HUMABIO)", FP6 call IST 4, http://www.humabioeu.org/, last accessed on 08/06/2009

[12] EC co-funded Specific Targeted Research Project (STREP), "Unobtrusive Authentication Using ACTIvity Related and Soft BIOmetrics (ACTIBIO)", FP7-ICT-2007-1, http://www.actibio.eu/, last accessed on 08/06/2009.

[13] Lorussi F., Scilingo E. P., Tesconi M., Tognetti A., De Rossi D., "Strain Sensing Fabric for Hand Posture and Gesture Monitoring", IEEE Transactions on Information Technology in Biomedicine, vol. 9, n. 3, pp. 372-381, 2005.

[14] Lorussi F., Rocchia W., Scilingo E. P., Tognetti A., De Rossi D., "Wearable Redundant Fabric-Based Sensors Arrays for reconstruction of Body Segment posture", IEEE Sensors Journal, vol. 4, n. 6, pp. 807$818,2004$.

[15] The Wacker Company, "The grades and properties of Elastosil LR liquid silicone rubber", http://www.wacker.com/ internet/webcache/de_DE/_Downloads/ EL_LR_Eigensch_en.pdf, last accessed on $17 / 03 / 2008$.

[16] Pioggia G., Ferro M., Di Francesco F., Dalle Mura G., De Rossi D., “An Architecture for High Efficiency Realtime Sensor and Actuator Data Processing", EUROSENSORS XIX, Barcelona, Spain, September 11th14th, 2005.

[17] The BioAPI Consortium, http://www.bioapi.org/, last accessed on 17/03/2008.

[18] Ross A.A., Nandakumar K., and Jain A. K., "Handbook of Multibiometrics", ser. Int. Series on Biometrics, Springer-Verlag New York, Inc. Secaucus, NJ, USA, Dec. 2006, vol. 6, ISBN 978-0-387-22296-7

[19] Jain A. K., "Biometric recognition: Q\&A", Nature, Vol. 449, pp. 38-40, September 2007.

[20] Kohonen T., "Self-Organization and Associative Memory", Springer, second edition, 1988 .

[21] Kohonen T., "Self-Organising Maps", Springer Series in Information Sciences, Vol. 30, Springer, Berlin, Heidelberg, New York, 2nd extended edition, 1997.

[22] Kinnebrock W., "Neural Networks", Munchen, Oldenburg Verlag, 1992. 